\title{
Dynamic non-classicality
}

\author{
Matthew Mandelkern
}

This is a postprint of an article published in the Australasian Journal of Philosophy, available online at https://www.tandfonline.com/doi/full/10.1080/00048402.2019.1624376, updated August 4, 2021

\begin{abstract}
I show that standard dynamic approaches to the semantics of epistemic modals invalidate the classical laws of excluded middle and non-contradiction, as well as the law of epistemic non-contradiction. I argue that these facts pose a serious challenge.
\end{abstract}

Keywords: dynamic semantics, epistemic modals, classical logic

\section{Introduction}

In the 1970s and 80s, formal semantics took a dynamic turn which aimed to capture the behaviour of dynamic features of natural language like anaphora resolution [Karttunen 1976; Heim 1982; Kamp 1981], presupposition projection [Karttunen 1974; Stalnaker 1974; Heim 1983], and epistemic modality [Veltman 1996; Groenendijk et al. 1996]. Our focus will be on the last of these: words like 'might' and 'must', on a broadly epistemic interpretation. The apparently dynamic features of epistemic modality is nicely illustrated by a contrast from Yalcin 2007. Compare:

a. \# Suppose it's raining and it might not be.

b. Suppose it's raining and for all we know it isn't.

Intuitively, 'It might not be raining' means roughly the same thing as 'For all we know, it's not raining'. But the two sentences in (1) sound very different: (1a) sounds incoherent, (1b) does not. One way to explain this is to hold that the information 
in the left conjunct in (1a)—-that it's raining-is dynamically incorporated into the interpretation of the 'might' in the right conjunct, so that (1a) ends up meaning something like: 'Suppose it's raining and it might (be raining and not be raining)'. This would straightforwardly account for contrasts like those in (1), and similar contrasts in a very wide variety of environments. ${ }^{1}$ Dynamic systems ${ }^{2}$ aim to account for effects like this—along with related phenomena involving anaphora and presupposition-by treating sentence meanings as functions which take a context to a new context, rather than as truth-conditions, as on static approaches.

The aim of this paper is to point out that these systems invalidate a wide variety of classical laws which are apparently valid. I argue that these facts pose a serious challenge for dynamic systems. ${ }^{3}$

\section{Classical principles}

The classical laws I will focus on are the following: (where $\varphi$ is a sentence and $\vDash \phi$ means that $\phi$ is a logical truth): ${ }^{4}$

(2) Law of Excluded Middle (EM): $\vDash(\varphi \vee \neg \varphi)$

(3) Meta-Language Non-Contradiction $\left(\mathrm{NC}_{m}\right):(\varphi \wedge \neg \varphi)$ is not consistent.

$$
\text { Object-Language Non-Contradiction }\left(\mathrm{NC}_{o}\right): \vDash \neg(\varphi \wedge \neg \varphi)
$$

1 See related examples in, for instance, Groenendijk et al. 1996; Aloni 2001; Dorr \& Hawthorne 2013; Yalcin 2015; Ninan 2018; Mandelkern 2019.

2 Of the kind I focus on here; DRT systems [Kamp 1981] as far as I know does not face similar challenges to those I raise here.

3 The challenge I raise here also extends to systems which replicate certain features of dynamic semantics in a static setting, for instance the system in Klinedinst \& Rothschild 2012, though I won't discuss that further.

4 I generally omit corner quotes for readability. 
Dynamic non-classicality

Thus EM says, for instance, that a sentence like (5) is always true:

(5) Either John came to the party or John didn't come to the party.

And $\mathrm{NC}_{m}$ says that (6) is never true, while $\mathrm{NC}_{o}$ says that the negation of (6) is always true.

(6) John came to the party and John didn't come to the party.

These laws are widely taken to be central in characterising the behaviour of disjunction, conjunction, and negation.

\section{Dynamic semantics}

$\mathrm{EM}, \mathrm{NC}_{o}$, and $\mathrm{NC}_{m}$ are all invalid in standard dynamic approaches to epistemic modals. I will illustrate this with a simple version of the influential system from Groenendijk et al. 1996. ${ }^{5}$ The points I make here extend, however, to every dynamic system I know of which includes epistemic modality ${ }^{6}$ except those which adopt static connectives, as I discuss in section 5.4. As I only discovered after this paper was first published, van Benthem 1996 already observed the failure of $\mathrm{NC}_{o}$, but as far as I know the point has not been subsequently discussed.

In this framework, a sentence $\varphi$ denotes not a proposition (as in static frameworks), but rather a context change potential $[\varphi]$ : a function from contexts (sets of possible worlds) to contexts, standardly written in post-fix notation (so that $c[\varphi]$

5 This comprises the semantics for atomic updates, negation, and conjunction of Heim 1983, together with the entries for disjunction from Groenendijk et al. 1996 and epistemic 'might' (abbreviated $\diamond$ ) from Veltman 1996.

6 Including prominent developments of Groenendijk et al. [1996]'s system, for instance Dekker 1993; Aloni 2001; Beaver 2001; Gillies 2001; Yalcin 2015; Gillies 2018; Goldstein 2019. 
is the result of applying $[\varphi]$ to $c$ ). The semantics is given recursively from a set At of atomic sentences $p, q, r \ldots$, a set of possible worlds $W$, and an interpretation function $I$ which assigns subsets of $W$ to elements of $A t$. Our language is the set which results from closing $A t$ under the one-place operators $\neg$ and $\diamond$ and two-place connectives $\wedge$ and $\vee$. For any context $c$ :

$$
\begin{aligned}
& \text { - } c[p]=\{w \in c: w \in I(p)\} \\
& \text { - } c[\varphi \wedge \psi]=c[\varphi][\psi] \\
& \text { - } c[\neg \varphi]=c \backslash c[\varphi] \\
& \text { - } c[\varphi \vee \psi]=c[\varphi] \cup c[\neg \varphi][\psi] \\
& \text { - } c[\vee \varphi]=\{w \in c: c[\varphi] \neq \varnothing\}
\end{aligned}
$$

This system aims to capture the dynamic behaviour of epistemic modals illustrated by sentences like (1a). Conjunction is treated as successive updating, first with the left conjunct, then with the right. And $\diamond \varphi$ is treated as a check of whether a context is consistent with $\varphi$. This means that, in a conjunction with the form $p \wedge \diamond(\neg p)$, the 'might' will check a context which has already been updated with $p$, and so this check will always fail. Such sentences are thus contradictions in this system, accounting for the infelicity of sentences like (1a) - and, the hope is, more generally accounting for the dynamic nature of epistemic modality.

In addition to these semantic entries, it will be helpful to define the following technical terms:

Idempotence: $\varphi$ is idempotent iff $\forall c: c[\varphi]=c[\varphi][\varphi]$ 
Dynamic non-classicality

Eliminativity: $\varphi$ is eliminative iff $\forall c: c[\varphi] \subseteq c$

Acceptance: $c$ accepts $\varphi$ iff $c=c[\varphi]$

Finally, we define entailment and consistency, following Veltman 1996:

Entailment: A sequence $\left\langle\varphi_{1}, \varphi_{2}, \ldots \varphi_{n}\right\rangle$ entails a sentence $\psi$, written $\varphi_{1}, \varphi_{2}, \ldots \varphi_{n} \vDash \psi$, iff $\forall c: c\left[\varphi_{1}\right]\left[\varphi_{2}\right] \ldots\left[\varphi_{n}\right]=c\left[\varphi_{1}\right]\left[\varphi_{2}\right] \ldots\left[\varphi_{n}\right][\psi]$.

Consistency: A sequence of sentences $\left\langle\varphi_{1}, \varphi_{2}, \ldots \varphi_{n}\right\rangle$ is consistent iff $\exists c: c\left[\varphi_{1}\right]\left[\varphi_{2}\right] \ldots\left[\varphi_{n}\right] \neq \varnothing$.

Thus for $\varphi$ to be a logical truth (written $\vDash \psi$ ) in this framework is for $\psi$ to be accepted by every context. It is important to note that this definition of entailment takes us far from the classical definitions in terms of preservation of truth, since in dynamic systems in general, truth does not play a central role. This means, for instance, that, rather than saying that $\varphi \vee \neg \varphi$ is true in every model, EM says that it is accepted in every context; likewise, instead of saying that $\varphi \wedge \neg \varphi$ is false in every model, $\mathrm{NC}_{m}$ says that it takes every context to the empty set. So in the context of dynamic semantics, these classical laws have a different interpretation than in static settings. ${ }^{7}$ However, the dynamic notion of entailment, and hence of logical truth, is meant to answer to roughly the same kind of intuitions that motivate static formulations: namely, the feeling that a given argument is a good one (or that a given sentence is always true/accepted). Hence the considerations that motivate these classical laws on their static interpretations (for instance, the intuition that sentences with the form $\varphi \vee \neg \varphi$ are always true/acceptable, and that $\varphi \wedge \neg \varphi$ never is) are also motivations for these laws as interpreted dynamically.

7 See Stokke 2014 for discussion of how we might get truth back into the picture. 


\section{Failures}

$\mathrm{EM}, \mathrm{NC}_{o}$, and $\mathrm{NC}_{m}$ are all invalid in this dynamic system. Consider first $\mathrm{NC}_{m}$. The question of whether $\mathrm{NC}_{m}$ is false in this system is the question of whether there is a context $c$ and sentence $\varphi$ such that $c[\varphi \wedge \neg \varphi] \neq \varnothing$. The answer is affirmative. The easiest way to see this is to note that, for any $c$ and $\varphi, c[\varphi \wedge \neg \varphi]=c[\varphi] \backslash c[\varphi][\varphi]$. Now if there is a sentence $\varphi$ in our system which is eliminative but fails to be idempotent, then there must be a $c$ such that $c[\varphi][\varphi]$ is a proper subset of $c[\varphi]$. Then it would follow that $c[\varphi] \backslash c[\varphi][\varphi] \neq \varnothing$, and thus that $c[\varphi \wedge \neg \varphi] \neq \varnothing$.

And indeed there are many sentences in our system which are eliminative but not idempotent. Here are two examples:

$$
\diamond p \wedge \neg p
$$

$$
(\diamond p \wedge \diamond q) \wedge(\neg p \vee \neg q)
$$

These are eliminative, since (from a simple induction on the length of formulas) all sentences in our system are eliminative: updating always results in discarding worlds from a context or leaving it unchanged. And these are non-idempotent. Consider a context $s=\left\{w, w^{\prime}\right\}$, with $w \in I(p), w \in I(q), w^{\prime} \notin I(p)$, and $w^{\prime} \notin I(q)$. Then $s[(7)]=\left\{w^{\prime}\right\}$, but $s[(7)][(7)]=\varnothing$. Likewise $s[(8)]=\left\{w^{\prime}\right\}$ but $s[(8)][(8)]=\varnothing$.

It thus follows that $(\varphi \wedge \neg \varphi)$ is consistent for some substitution instances of $\varphi$; in particular, it will be consistent when we put in either (7) or (8) for $\varphi$. More concretely, consider $s[(\diamond p \wedge \neg p) \wedge \neg(\diamond p \wedge \neg p)]$. By our semantic rules, we have:

$$
s[(\diamond p \wedge \neg p) \wedge \neg(\diamond p \wedge \neg p)]=
$$


Dynamic non-classicality

$$
\begin{gathered}
s[(\diamond p \wedge \neg p)][\neg(\diamond p \wedge \neg p)]= \\
s[\diamond p][\neg p][\neg(\diamond p \wedge \neg p)]= \\
\left\{w^{\prime}\right\}[\neg(\diamond p \wedge \neg p)]= \\
\left\{w^{\prime}\right\} \backslash\left(\left\{w^{\prime}\right\}[\diamond p][\neg p]\right)= \\
\left\{w^{\prime}\right\} \backslash \varnothing=\left\{w^{\prime}\right\}
\end{gathered}
$$

Thus $(\diamond p \wedge \neg p) \wedge \neg(\diamond p \wedge \neg p)$ fails to take every context to the empty set, and thus is consistent in the present system. Similar reasoning will show that $s[((\diamond p \wedge \diamond q) \wedge(\neg p \vee \neg q)) \wedge \neg((\diamond p \wedge \diamond q) \wedge(\neg p \vee \neg q))]=\left\{w^{\prime}\right\}$, and thus that $((\diamond p \wedge$ $\diamond q) \wedge(\neg p \vee \neg q)) \wedge \neg((\diamond p \wedge \diamond q) \wedge(\neg p \vee \neg q))$, too, is consistent. Thus $\mathrm{NC}_{m}$ is false in our system: not every sentence of the form $(\varphi \wedge \neg \varphi)$ is inconsistent.

Given the failure of $\mathrm{NC}_{m}$, it is straightforward to show the failure of $\mathrm{NC}_{o}$. Our definition of negation says that $c[\neg \varphi]=c \backslash c[\varphi]$; it follows that if $\varphi$ is consistent, then there is a context $c$ such that $c[\varphi] \neq \varnothing$, and thus that, given eliminativity, $c \backslash c[\varphi] \neq c$, and thus that there is a context $c$ which fails to accept $\neg \varphi$. From the fact that $(\diamond p \wedge \neg p) \wedge \neg(\diamond p \wedge \neg p)$ and $((\diamond p \wedge \diamond q) \wedge(\neg p \vee \neg q)) \wedge \neg((\diamond p \wedge \diamond q) \wedge(\neg p \vee \neg q))$ are consistent it thus follows that their negations are not accepted by every context, and thus are not logical truths. Thus $\mathrm{NC}_{o}$ is invalid in our system: not every sentence of the form $\neg(\varphi \wedge \neg \varphi)$ is a logical truth.

As a concrete example, consider the result when we update $s$ with $\neg((\diamond p \wedge \neg p) \wedge$ $\neg(\diamond p \wedge \neg p)) . \quad s[\neg((\diamond p \wedge \neg p) \wedge \neg(\diamond p \wedge \neg p))]=s \backslash s[(\diamond p \wedge \neg p) \wedge \neg(\diamond p \wedge \neg p)]$. Since we know the latter term is $\left\{w^{\prime}\right\}$, the whole expression is equal to $\{w\}$, which 
is not equal to $s$. Thus $s$ fails to accept $\neg((\diamond p \wedge \neg p) \wedge \neg(\diamond p \wedge \neg p))$. Likewise, $s$ will not accept $\neg(((\diamond p \wedge \diamond q) \wedge(\neg p \vee \neg q)) \wedge \neg((\diamond p \wedge \diamond q) \wedge(\neg p \vee \neg q))){ }^{8}$

Finally, EM will not be valid in our system: $(\varphi \vee \neg \varphi)$ is not a logical truth. To see this abstractly, note that $c[\varphi \vee \neg \varphi]=c[\varphi] \cup c[\neg \varphi][\neg \varphi]$. Given that our system is eliminative, $c[\varphi] \cup c[\neg \varphi][\neg \varphi]$ will always be equal to $c$ iff $\neg \varphi$ is idempotent, since then $c[\varphi] \cup c[\neg \varphi][\neg \varphi]=c[\varphi] \cup c[\neg \varphi]=c[\varphi] \cup(c \backslash c[\varphi])=c$. But there are many sentences $\varphi$ in our system such that $\neg \varphi$ fails to be idempotent, and thus EM is not valid.

To find such a $\varphi$, note that double negation elimination is valid in our system: $\forall \varphi:[\neg \neg \varphi]=[\varphi]$, since $\forall c: c[\neg \neg \varphi]=c \backslash c[\neg \varphi]=c \backslash(c \backslash c[\varphi])=c[\varphi]$ (again, the last step follows because of eliminativity). And thus the negation of (7), namely $\neg(\diamond p \wedge \neg p)$, will have a negation equivalent to (7), and thus will have a negation which is not idempotent. So let $\varphi=\neg(\diamond p \wedge \neg p)$, and now consider the context $s$ from our previous example, updated with our sentence $\neg(\diamond p \wedge \neg p) \vee \neg(\neg(\diamond p \wedge \neg p))$ :

$$
\begin{gathered}
s[\neg(\diamond p \wedge \neg p) \vee \neg(\neg(\diamond p \wedge \neg p))]= \\
s[\neg(\diamond p \wedge \neg p)] \cup s[\neg(\neg(\diamond p \wedge \neg p)][\neg(\neg(\diamond p \wedge \neg p)]= \\
(s \backslash s[\diamond p \wedge \neg p]) \cup s[\diamond p \wedge \neg p][\diamond p \wedge \neg p]=
\end{gathered}
$$

8 A close corollary of these facts concerns conditionals with the form $\varphi \rightarrow \varphi$. Given the standard dynamic semantics for the conditional from Groenendijk et al. 1996, $c$ accepts $\varphi \rightarrow \psi$ iff $c[\varphi]$ accepts $\psi$. Since $c[\varphi] \neq c[\varphi][\varphi]$ for non-idempotent sentences like those we are looking at here, $\varphi \rightarrow \varphi$ will not always be accepted. 
Dynamic non-classicality

$$
\begin{gathered}
\left(s \backslash\left\{w^{\prime}\right\}\right) \cup\left\{w^{\prime}\right\}[\diamond p \wedge \neg p]= \\
\{w\} \cup \varnothing=\{w\} \neq s
\end{gathered}
$$

Thus $s$ does not accept $(\varphi \vee \neg \varphi)$ when we let $\varphi=\neg(\diamond p \wedge \neg p)$. And so $(\varphi \vee \neg \varphi)$ is not a logical truth for all substitution instances of $\varphi$, and thus EM is false in our system. (Further counterexamples to EM are again straightforward to produce, for instance by substituting the negation of (8) for $\varphi$.)

\subsection{Epistemic non-contradiction}

A final observation. It has been frequently claimed that Epistemic Non-Contradiction holds in our system (for instance in Gillies 2001; von Fintel \& Gillies 2007; Yalcin 2012 2015):

(9) Epistemic Non-Contradiction (ENC): For all $\varphi,(\varphi \wedge \diamond \neg \varphi)$ is not consistent.

But it is easy to see that $\mathrm{ENC}$ is equivalent to $\mathrm{NC}_{m}$. The key point is that for any $c$ and $\varphi$ in our system, $c[\diamond \varphi]=\varnothing$ just in case $c[\varphi]=\varnothing$. Thus it follows that $(\varphi \wedge \neg \varphi)$ is inconsistent for all $\varphi$ if and only if $(\varphi \wedge \diamond \neg \varphi)$ will be as well. The fact that $\mathrm{NC}_{m}$ is invalid thus immediately entails that ENC is invalid. This fact is of independent interest (though I will not explore it in depth here). And dialectically, it suggests that 
the failure of $\mathrm{NC}_{m}$ (and thus of its sibling $\mathrm{NC}_{o}$ ) in our dynamic system has not been appreciated. ${ }^{9}$

For a concrete counterexample to ENC, we need only substitute (7) or (8) for $\varphi$. For instance, consider $(\diamond p \wedge \neg p) \wedge \diamond(\neg(\diamond p \wedge \neg p))$, and consider again the context $s$ from above:

$$
\begin{gathered}
s[(\diamond p \wedge \neg p) \wedge \diamond(\neg(\diamond p \wedge \neg p))]= \\
s[\diamond p \wedge \neg p][\diamond(\neg(\diamond p \wedge \neg p))]= \\
\left\{w^{\prime}\right\}[\diamond(\neg(\diamond p \wedge \neg p))]
\end{gathered}
$$

The result of updating $\left\{w^{\prime}\right\}$ with $\diamond(\neg(\diamond p \wedge \neg p))$ depends on whether the result of updating $\left\{w^{\prime}\right\}$ with $\neg(\diamond p \wedge \neg p)$ is empty. It is not:

$$
\begin{gathered}
\left\{w^{\prime}\right\}[\neg(\diamond p \wedge \neg p)]= \\
\left.\left\{w^{\prime}\right\} \backslash\left(\left\{w^{\prime}\right\}[\diamond p][\neg p)\right]\right)= \\
\left\{w^{\prime}\right\} \backslash \varnothing=\left\{w^{\prime}\right\}
\end{gathered}
$$

9 The failure of ENC is implicit in a handout of Klinedinst \& Rothschild [2014] which notes that negated epistemic contradictions are not tautologies. 
Dynamic non-classicality

Thus $\left\{w^{\prime}\right\}[\neg(\diamond p \wedge \neg p)]$ is non-empty, and thus $\left\{w^{\prime}\right\}[\diamond(\neg(\diamond p \wedge \neg p))]=\left\{w^{\prime}\right\}$. And so $s[(\diamond p \wedge \neg p) \wedge \diamond(\neg(\diamond p \wedge \neg p))]=\left\{w^{\prime}\right\}$, not $\varnothing$. Thus ENC is false in our system.

\section{Possible responses}

What should we make of these facts?

\subsection{Embrace the result}

The most daring response on behalf of dynamic treatments of epistemic modality would be to embrace these results: to argue that only limited variants of $\mathrm{EM}, \mathrm{NC}_{o}$, and $\mathrm{NC}_{m}$ are in fact true, and that these principles fail precisely where dynamic semantics predicts them to fail.

Certain failures of these laws in dynamic systems, in particular systems enriched to handle anaphora, might warrant this response. In such systems (for instance the system of Groenendijk \& Stokhof 1991), these laws can fail in sentences without epistemic modals, provided that pronouns and quantifiers are co-indexed in a certain way. But since there are no completely transparent ways to determine when quantifiers and pronouns are and are not co-indexed in natural language (we do not have numeric subscripts on our words), it is not altogether obvious what empirical purchases these failures have; and so, while there is perhaps no evidence for these failures, it is hard to find positive evidence that these predictions are wrong.

But I do not see any prospects for a defense along these lines when we focus on the failures of these laws that I have pointed to above. For when it comes to these failures, it seems clear what the intended, unambiguous translation of these sentences into natural language amounts to (once we fix arbitrarily on meanings for 
our atomic sentences); and it is clear that the predicted failures of $\mathrm{EM}, \mathrm{NC}_{o}$, and $\mathrm{NC}_{m}$ do not match intuitions about the corresponding sentences in natural language. To make this point, let $p=$ 'Paul is sick', and let $q=$ 'Mark is sick'. Then (7) will be translated into natural language as (10):

(10) Paul might be sick and he isn't.

And (8) will be translated as (11a) (or more succinctly as (11b)):

(11) a. Paul might be sick and Mark might be sick, and either Paul isn't sick or Mark isn't sick.

b. Paul might be sick and Mark might be sick, but one of them isn't sick.

Now let us ask whether the predicted failures of $\mathrm{EM}, \mathrm{NC}_{o}$, and $\mathrm{NC}_{m}$ match intuition. Focus on (11), since (10) is itself quite weird to begin with. The predicted failure of $\mathrm{NC}_{m}$ that we get from (11) is in (12):

(12) Paul might be sick and Mark might be sick, but one of them isn't sick; and it's not the case that (Paul might be sick and Mark might be sick, but one of them isn't sick).

Its complexity notwithstanding, (12) seems to be perfectly inconsistent, like any contradiction of the form $(\varphi \wedge \neg \varphi)$. Likewise, the negation of (12) in (13) seems to be trivially true:

(13) It's not the case that: (Paul might be sick and Mark might be sick, but one of them isn't sick; and it's not the case that (Paul might be sick and Mark might be sick, but one of them isn't sick)). 
Dynamic non-classicality

But our dynamic system predicts that (12) is consistent, and that (13) is not a logical truth. These predicted failures of $\mathrm{NC}_{m}$ and $\mathrm{NC}_{o}$ thus do not by any means seem to match intuition.

Nor are matters better when we turn to EM. Consider (14):

(14) Either Paul might be sick and Mark might be sick, but one of them isn't sick; or it's not the case that (Paul might be sick and Mark might be sick, but one of them isn't sick).

Again, complexity notwithstanding, (14) seems to be a logical truth, that is trivially true, as much as any disjunction of the form $(\varphi \vee \neg \varphi)$. But this is again contrary to the predictions of our theory, which says that (14) is not a logical truth.

$\mathrm{EM}, \mathrm{NC}_{o}$, and $\mathrm{NC}_{m}$ are by no means sacrosanct. We may find reasons to criticise them, for instance, having to do with semantic paradoxes, vagueness, or with puzzles about indexicality. And constrained failures of these laws in a formal system may be harmless or even desirable, if those failures conform, or at least fail to conflict, with intuition about natural language. But, in the present case, the specific counterexamples to these laws predicted by our system $d o$ conflict with intuition about the corresponding natural language sentences. Thus I do not think there is any prospect for a defender of dynamic semantics simply to embrace these results.

\subsection{Adopt a different notion of entailment}

A second avenue that defenders of dynamic semantics might pursue is to adopt a different notion of entailment and consistency. For instance, spelling out $\mathrm{NC}_{m}$ in 
terms of Groenendijk et al. [1996]'s notion of coherence, rather than consistency, changes the picture:

$$
\mathrm{NC}_{m} *:(\varphi \wedge \neg \varphi) \text { is not coherent. }
$$

Where coherence is defined as follows:

Coherence: $\varphi$ is coherent iff $\exists c: c \neq \varnothing \wedge c[\varphi]=c$.

$\mathrm{NC}_{m}{ }^{*}$, by contrast to $\mathrm{NC}_{m}$, is valid in our system. To see this, assume that $c=c[\varphi \wedge \neg \varphi]=c[\varphi] \backslash c[\varphi][\varphi]$. Given eliminativity, we have two options: $c[\varphi]=c$ or $c[\varphi] \subsetneq c$. If the latter, then it follows that $c[\varphi] \backslash c[\varphi][\varphi] \subsetneq c$. But we already know that $c[\varphi] \backslash c[\varphi][\varphi]=c$; contradiction. So $c[\varphi]=c$. But then of course $c[\varphi][\varphi]$ is also $c$, and so $c[\varphi] \backslash c[\varphi][\varphi]=c \backslash c=\varnothing$ and so $c=\varnothing$. And so $(\varphi \wedge \neg \varphi)$ is only accepted by the empty context; and so it is not coherent.

But this is little help. The first, milder, issue is that consistency can be bootstrapped to coherence with just the help of an epistemic modal: that is, even though sentences with the form $(\varphi \wedge \neg \varphi)$ are not coherent, sentences of the form $\diamond(\varphi \wedge \neg \varphi)$ will be not only consistent but also coherent (the same substitution instances as above witness this; if there are non-empty contexts $s$ and sentences $(\varphi \wedge \neg \varphi)$ such that $s[(\varphi \wedge \neg \varphi)] \neq \varnothing$, then it follows immediately that $s[\diamond(\varphi \wedge \neg \varphi)]=s)$. But this result seems just as bad as the prediction that $(\varphi \wedge \neg \varphi)$ is coherent; the fact that $(\varphi \wedge \neg \varphi)$ is incoherent is little comfort if close variations like this remain coherent (variations are easy to come by; for instance $\exists x(\varphi(x) \wedge \neg \varphi(x))$ will be coherent if we adopt Groenendijk et al. 1996's semantics for $\exists$ ).

The second, more important, issue is that this response does nothing to change our predictions about EM or $\mathrm{NC}_{o}$. Adopting a different notion of entailment won't 
Dynamic non-classicality

save $\mathrm{EM}$ or $\mathrm{NC}_{o}$, because all the main dynamic theories of entailment coincide when it comes to their definitions of logical truth. ${ }^{10}$ And so even if we could (imprudently) convince ourselves to be satisfied with $\mathrm{NC}_{m}$ * in place of $\mathrm{NC}_{m}$, we would remain in serious trouble from the failures of $\mathrm{EM}$ and $\mathrm{NC}_{o}$.

\subsection{Insist on idempotence}

The final two responses worth considering acknowledge the seriousness of the failures of $\mathrm{NC}_{m}, \mathrm{NC}_{o}$, and $\mathrm{EM}$ in our system, and propose to change the system to avoid these failures, while remaining in a broadly similar framework. The first of these responses goes as follows. As we saw above, all the failures of our three principles stem from failures of idempotence; in a fully idempotent fragment, our entries for conjunction and negation suffice to validate $\mathrm{NC}_{o}$ and $\mathrm{NC}_{m}$; and, assuming the fragment remains eliminative, our entries for disjunction and negation suffice to validate EM. One option to take is thus to eliminate idempotence from our system.

There are two different ways to do this. One is global: ensure that no operators are added to the language which can combine with existing parts of the language to create non-idempotent sentences. This would simply rule out Veltman [1996]'s semantics for $\diamond$. A different approach is local: rule out by fiat non-idempotent sentences (but still allow sentences with $\diamond$, provided they are idempotent). This local approach is suggested for different reasons in Yalcin 2015, building on Klinedinst \& Rothschild 2014.

The local approach strikes me as a non-starter, for it rules out sentences with the form of (8), which, as we saw above, are non-idempotent, but which translate

10 This is so, for instance, of all the definitions of entailment given in Veltman 1996. 
to perfectly felicitous sentences of natural language (like (11)). Any account of epistemic modals which predicts a sentence like (8) to be inconsistent or ill-formed is the wrong account. ${ }^{11}$

The global approach, by contrast, is more plausible. However, by ruling out Veltman's $\diamond$, this approach would undermine one of the major motivations for dynamic semantics_-providing a unified treatment of the dynamics of anaphora, presupposition, and epistemic modality. If this is the right route to take, it requires a major shift in thinking about the aims and limitations of dynamic semantics, and how to model epistemic modals in that framework.

Even then, there remains a rather abstract consideration which tells against an approach like this. Any theory which incorporates the entries for conjunction, disjunction, and negation above will incur the debt of adopting further stipulations to ensure that the theory validates the classical laws we are discussing (for instance, by ruling out failures of idempotence). Standard static Boolean approaches, by contrast, validate these three principles without having to appeal to further stipulations beyond the standard semantics for the connectives. This strikes me as a major theoretical benefit of static approaches over their dynamic competitors.

\subsection{Adopt different connectives}

In light of this last consideration, it is worth asking, finally, whether dynamic semanticists can have what static semantics gets so easily—a semantics for connectives

11 Klinedinst \& Rothschild 2014 proposes an interesting modification of a semantic system like ours which rules out some but not all idempotence. Essentially on their system, local context updates are always repeated until they reach a fixed point. This helps with the issues around non-contradiction without falling into the present pitfall. It is, however, no help at all in avoiding the issues for excluded middle. 
Dynamic non-classicality

which immediately validates $\mathrm{EM}, \mathrm{NC}_{o}$, and $\mathrm{NC}_{m}$, without further stipulations about admissible vocabulary in the language.

Indeed they can, or at least nearly so. The following semantics for conjunction, from Veltman 1996, together with the standard negation from above, guarantees the validity of $\mathrm{NC}_{o}$ and $\mathrm{NC}_{m}$, no matter how the language is extended:

$$
\text { - } c[\varphi \wedge \psi]=c[\varphi] \cap c[\psi]
$$

This is because, in this system, $\forall c: c[\neg(\varphi \wedge \neg \varphi)]=c \backslash(c[\varphi] \cap c[\neg \varphi])=c \backslash(c[\varphi] \cap$ $(c \backslash c[\varphi]))=c$, and so every context accepts $\neg(\varphi \wedge \neg \varphi)$. Likewise, $(\varphi \wedge \neg \varphi)$ will take every context to $\varnothing$, since $\forall c: c[\varphi \wedge \neg \varphi]=c[\varphi] \cap(c \backslash c[\varphi])=\varnothing$. So $\mathrm{NC}_{o}$ and $\mathrm{NC}_{m}$ will be valid, no matter how the system is extended.

Likewise, if we adopt this disjunction from Veltman 1996:

$$
\text { - } c[\varphi \vee \psi]=c[\varphi] \cup c[\psi]
$$

then EM will be guaranteed to be valid for any extension of the fragment, with the important caveat that the system must be extended in a way which always preserves eliminativity. In that case we will have that $\forall c: c[\varphi \vee \neg \varphi]=c[\varphi] \cup(c \backslash c[\varphi])$, which will be $c$, thanks to eliminativity. This is not yet quite as general as we would want it to be, but it is a major improvement on the present situation.

So should we reject the standard dynamic system in favor of these entries? There is reason to hesitate. First, although these entries validate $\mathrm{NC}_{o}$ and $\mathrm{NC}_{m}$, they still face a closely related problem. These entries validate $\mathrm{NC}_{o}$ and $\mathrm{NC}_{m}$ because, unlike the standard entry for conjunction, conjunction is treated as set intersection rather than consecutive update. But consecutive assertions, of course, are still treated as 
consecutive updates. And so, even though this approach predicts that all sentences of the form $(\varphi \wedge \neg \varphi)$ are inconsistent, it predicts that sequential assertions of the form $\langle\varphi, \neg \varphi\rangle$ are perfectly consistent (the same substitution instances as above witness this, for precisely the same reason). This prediction seems just as problematic as failing to validate $\mathrm{NC}_{o}$ or $\mathrm{NC}_{m}$, and so it is not really clear that this approach solves our problem. ${ }^{12}$

Second, and equally seriously, these entries lose much of what is attractive and interesting about the standard dynamic entries: their ability to capture the characteristically dynamic intrasentential behaviour of epistemic modality, presupposition, and anaphora, which, after all, is the whole point of dynamic semantics. The entries under consideration validate none of the intrasentential patterns which have been used to motivate dynamic semantics, and which have been claimed as successes for dynamic semantics. I will mention just two examples here.

First, although I have shown that Epistemic Non-Contradiction is not valid in general in the standard system laid out above, it is valid in that system when substitution for $\varphi$ is restricted to the non-modal fragment of the system (that is, $(\varphi \wedge \diamond \neg \varphi)$ will be inconsistent provided $\varphi$ does not contain $\diamond)$. This remains a success of dynamic semantics, albeit a more limited one than has been claimed. But this success would be entirely lost if we adopted the entry for conjunction under consideration, on which epistemic contradictions of all kinds would be consistent.

Second, there are apparent asymmetries in how we process conjunctions containing anaphora and presupposition; for instance, 'A man walked in and he sat down' is processed quite differently from 'He sat down and a man came in'. Likewise 'France

12 Though these sequences will be incoherent, and maybe some mileage can be got out of this fact to explain what is wrong with them. 
Dynamic non-classicality

has a king, and the king of France is bald' strikes us as being quite different from 'The king of France is bald, and France has a king'. The standard dynamic entries are designed to predict precisely these asymmetries. By contrast, the entries under consideration here are commutative: they do not predict these order asymmetries. So going this way would sacrifice much of what is attractive not only in dynamic treatments of epistemic modality, but also in dynamic treatments of anaphora and presupposition.

\section{Conclusion}

The challenge which these considerations raise, and which I leave open here, is whether (and if so how) a dynamic treatment of a fragment including epistemic modals can capture its characteristic dynamic patterns without simultaneously invalidate classical patterns which we have every reason to preserve.

\section{Acknowledgements}

Many thanks to Paul Dekker, Anthony Gillies, Simon Goldstein, Daniel Rothschild, Ginger Schultheis, and two anonymous referees for this journal for very helpful comments and discussion.

\section{References}

Aloni, Maria D. 2001. Quantification under conceptual covers. Amsterdam: University of Amsterdam dissertation. 
Beaver, David. 2001. Presupposition and assertion in dynamic semantics. CSLI Publications: Stanford, CA. http://codenlp.ru/wp-content/uploads/2014/01/ Presuppozitsii-1-ENG.pdf.

van Benthem, J. 1996. Exploring logical dynamics. Stanford, CA: Center for the Study of Language and Information.

Dekker, Paul. 1993. Transsentential meditations: Ups and downs in dynamic semantics: University of Amsterdam dissertation.

Dorr, Cian \& John Hawthorne. 2013. Embedding epistemic modals. Mind 122(488). 867-913. http://dx.doi.org/10.1093/mind/fzt091.

von Fintel, Kai \& Anthony Gillies. 2007. An opinionated guide to epistemic modality. In Tamar Gendler \& John Hawthorne (eds.), Oxford studies in epistemology 2, 32-62. Oxford.

Gillies, Anthony. 2001. A new solution to Moore's paradox. Philosophical Studies 105. 237-250.

Gillies, Anthony S. 2018. Updating data semantics. Mind http://dx.doi.org/10.1093/ $\mathrm{mind} / \mathrm{fzy} 008$.

Goldstein, Simon. 2019. Generalized update semantics. Mind 128(511). 795-835. http://dx.doi.org/10.1093/mind/fzy076.

Groenendijk, Jeroen \& Martin Stokhof. 1991. Dynamic predicate logic. Linguistics and Philosophy 14(1). 39-100. http://dx.doi.org/10.1007/BF00628304.

Groenendijk, Jeroen, Martin Stokhof \& Frank Veltman. 1996. Coreference and modality. In Shalom Lappin (ed.), Handbook of contemporary semantic theory, 179-216. Oxford: Blackwell. 
Dynamic non-classicality

Heim, Irene. 1982. The semantics of definite and indefinite noun phrases: University of Massachusetts, Amherst dissertation. https://semanticsarchive.net/Archive/ jA2YTJmN/Heim\%20Dissertation\%20with\%20Hyperlinks.pdf.

Heim, Irene. 1983. On the projection problem for presuppositions. In Michael Barlow, Daniel P. Flickinger \& Nancy Wiegand (eds.), The west coast conference on formal linguistics (WCCFL), vol. 2, 114-125. Stanford Stanford University Press. http://dx.doi.org/10.1002/9780470758335.ch10.

Kamp, Hans. 1981. A theory of truth and semantic representation. In J. Groenendijk et al. (ed.), Formal methods in the study of language, Mathematisch Centrum.

Karttunen, Lauri. 1974. Presupposition and linguistic context. Theoretical Linguistics 1(1-3). 181-194. http://dx.doi.org/10.1515/thli.1974.1.1-3.181.

Karttunen, Lauri. 1976. Discourse referents. In Syntax and semantics: Notes from the linguistic underground, vol. 7, Academic Press.

Klinedinst, Nathan \& Daniel Rothschild. 2012. Connectives without truth tables. Natural Language Semantics 20(2). 137-175.

Klinedinst, Nathan \& Daniel Rothschild. 2014. Epistemic contradictions: why idempotence is hygienic. Handout.

Mandelkern, Matthew. 2019. Bounded modality. The Philosophical Review 128(1). 1-61. http://dx.doi.org/10.1215/00318108-7213001.

Ninan, Dilip. 2018. Quantification and epistemic modality. The Philosophical Review 127(2). 433-485. http://dx.doi.org/10.1215/00318108-6973010.

Stalnaker, Robert. 1974. Pragmatic presuppositions. In Milton K. Munitz \& Peter Unger (eds.), Semantics and philosophy, 197-213. New York: New York University Press. http://dx.doi.org/10.1093/0198237073.003.0003. 
Stokke, Andreas. 2014. Truth and context change. Journal of Philosophical Logic 43(1). 33-51. http://dx.doi.org/10.1007/s10992-012-9250-6.

Veltman, Frank. 1996. Defaults in update semantics. Journal of Philosophical Logic 25(3). 221-261.

Yalcin, Seth. 2007. Epistemic modals. Mind 116(464). 983-1026. http://dx.doi.org/ 10.1093/mind/fzm983.

Yalcin, Seth. 2012. Dynamic semantics. In Gillian Russell \& Delia Graff Fara (eds.), Routledge companion to philosophy of language, Routledge.

Yalcin, Seth. 2015. Epistemic modality de re. Ergo 2(19). 475-527.

Matthew Mandelkern

All Souls College

Oxford, OX1 4AL

United Kingdom

matthew.mandelkern@all-souls.ox.ac.uk 